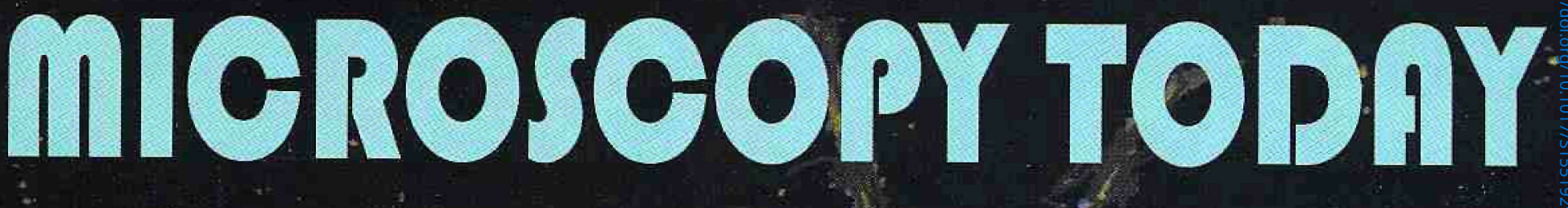

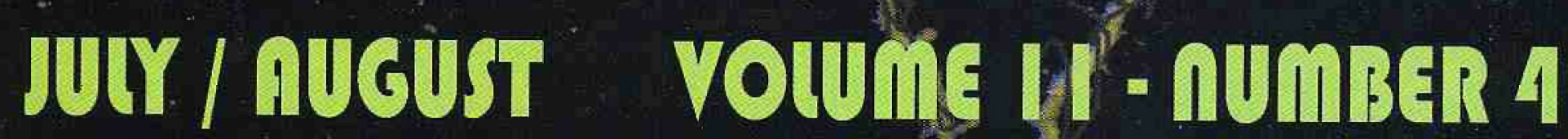

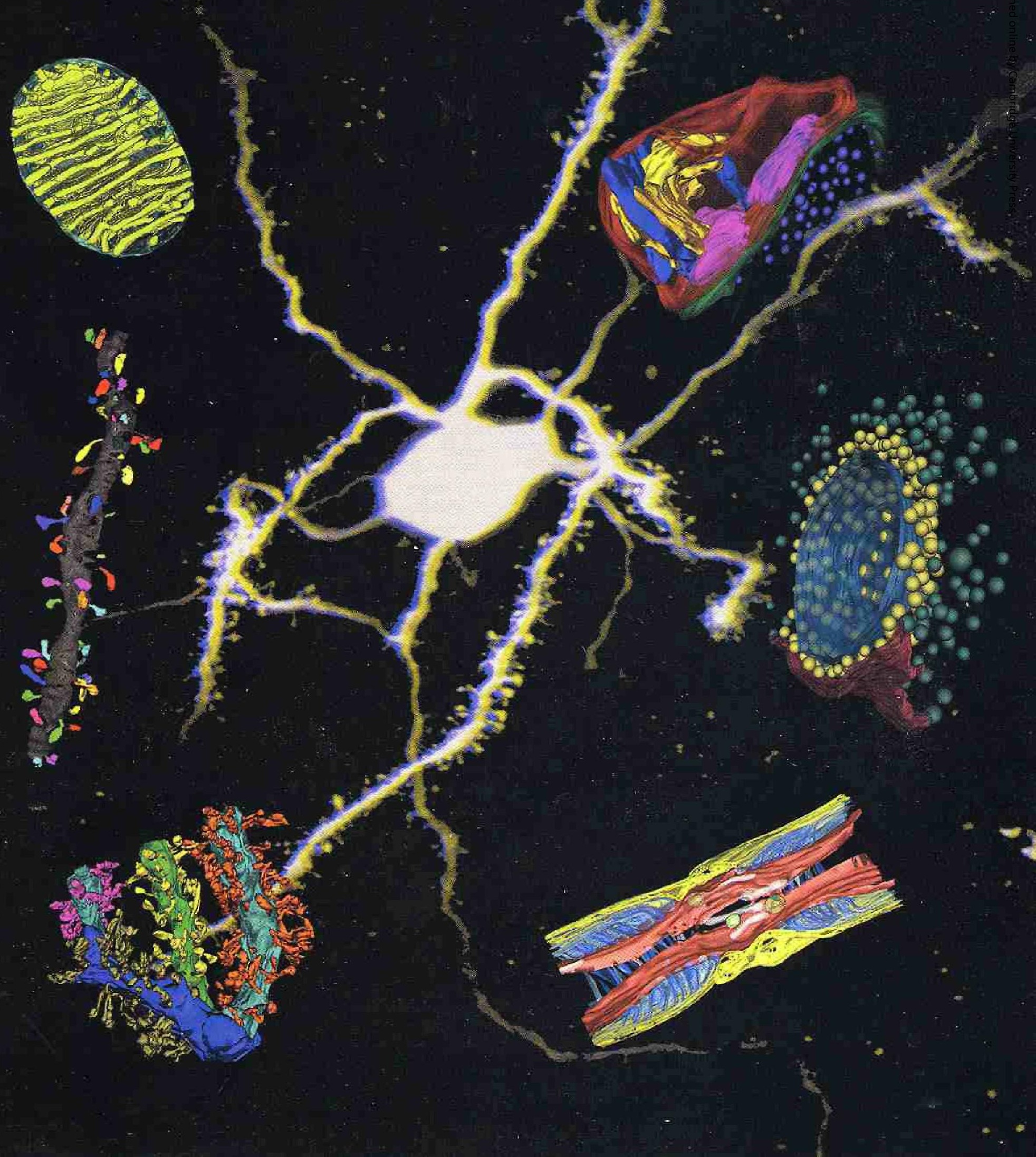




\section{Taking Correlative Microscopy to a New Level}

Stephen W. Carmichael, ${ }^{1}$ Mayo Clinic carmichael.stephen@mayo.edu

We are all familiar with the concept of correlating an image acquired by light microscopy (LM) with one obtained by transmission electron microscopy (TEM). This allows us to take advantage of the "wide angle" view of LM and the high resolution of TEM. Correlative microscopy has been taken to a new level by Alvin Lin and Cynthia Goh who have designed a clever device. ${ }^{2}$ This device allows repetitive correlative microscopy between TEM and atomic force microscopy (AFM).

The device is a novel sample holder that permits AFM to be performed on specimens mounted on TEM grids. This holder allows for the first time, AFM and TEM images on exactly the same area of the specimen. Whereas previous studies have correlated TEM with scanning probe microscopy (which includes AFM), reliable and repetitive examination of the same region has not been possible. The "trick" in the specimen holder designed by Lin and Goh was to secure a carbon-coated TEM index grid with plastic strips that are in turn secured with metal clips. These are mounted on a steel base that is secured magnetically to an AFM piezo scanner. The entire holder is sufficiently small to easily fit into an AFM. To allow reproducible relocation of specific areas in the AFM, indexed TEM grids were used and visualized with a CCD camera.

As proof of concept, Lin and Goh examined the structure of particular abnormal collagen fibrils, known as fibrous long spacing collagen. Fibers were precipitated in vitro onto a carbon-coated TEM indexed grid and then imaged by contact AFM and TEM.
Images were obtained of the same area (6 microns on a side) by both techniques. Then the specimens were negatively stained with phosphotungstic acid (PTA) and imaged again in both microscopes. This precise repetitive correlative microscopy by TEM and AFM is a tour de force.

In addition to this technical achievement, this study answered an important question about imaging collagen fibers (at least this abnormal type, bands in native collagen are much smaller, making correlative microscopy more difficult), specifically regarding the periodicity of the fiber. The role of PTA in negatively stained fibers has been controversial. Did the PTA fill in spaces between elevations on the fiber, or did it alter contrast by depositing on the substrate around the fiber? Lin and Goh were able to definitively demonstrate that elevated ridges that could be measured in the topographical data from the AFM corresponded to the dark regions seen by TEM, both before and after PTA staining. Additionally, measurements from AFM showed that the elevation changed by approximately the diameter of the PTA anion after staining. Therefore, dark and light features that give collagen its characteristic periodicity in TEM is due to the material in the fibril, and is not due to PTA. This could only be determined by imaging the exact same area repeatedly with the TEM and AFM.

It will be exciting to see what other interesting questions can be settled using this advance in correlative microscopy!

\section{References}

1. The author gratefully acknowledges Dr. M.Cynthia Goh for reviewing this article.

2. Lin, A.C., and M.C. Goh, A novel sample holder allowing atomic force microscopy on transmission electron microscopy grids: Repetitive, direct correlation between AFM and TEM images, J. Microscopy 205 205-208, 2002.

\section{InDEX or MNHIES}

Taking Correlative Microscopy to a New Level Stephen W. Carmichael, Mayo Clinic

Imaging of Big and Messy Biological Structures Using Electron Tomography.

Gina E. Sosinsky and Maryann E. Martone, University of California

A New Capability for Light Microscopes: Mid Infrared Molecular

Analysis Alan J. Rein, Sens/R Technologies

Brightfield Illumination of Large Field Sizes Theodore M. Clarke, Metallurgical Failure Analysis Consultant

Special Topic: Advanced Basics of Immunostaining and Antigen

Retrieval W. Gray (Jay) Jerome, Vanderbilt University

How To Get Some Action (In Photoshop) Jerry Sedgewick, University of Minnesota

Macro Imaging with Digital Cameras Bryan Bumett, Nyoptics, Inc./Meixa Tech and Steven Blaauw, Nyoptics, inc.

Rapid Preparation of a Polymer Fiber and a Free-Standing Polymer

Film for Cross-Sectional Microtomy

Andreas Taubert, University of Basel, and James H. Ferris, and Karen 1. Winey University of Pennsylvania

M\&M 2002: Core Facility Management Session: Maintaining Major

Equipment in the Core Microscopy Facility . Session Chair: Debby Sherman, Purdue University

Making Your Own Molds For The EM Lab Mary Mager, University of British Columbia

Vascular Corrosion Casting Fred E. Hossier, East Tennessee State University

Benzyldimethylamine (BDMA): Catalyst of Choice with Epoxy

Embedding Media José A. Mascorro, Tulane University Health Sciences Center
The Most Likely Sources of EDX Copper Peaks in Samples Run by TEM Paul Beauregard, Chemist and Electron Microscopist

NetNotes. 47

Industry News. 49

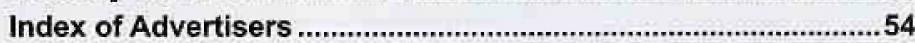
,

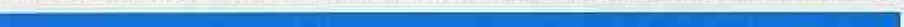

\section{ABOUT THE OOVER}

\section{Gina E. Sosinsky and Maryann E. Martone} Univ. of California

This composite image illustrates several examples of tomographic reconstructions of neuronal compartments. The center image is a projection through an optical series recorded on a 2-photon microscope of a medium spiny neuron from the nucleus accumbens of the mouse filled with Lucifer Yellow. The images surrounding the spiny neuron are reconstructions using single axis electron tomography and then surface rendered. The sizes of these structures range from $40 \mathrm{~nm}$ to $20 \mu \mathrm{m}$. Starting in the upper left hand corner and proceeding clockwise is a mitochondria, a synaptic complex from the rat hippocampus, a synaptic complex from the frog vestibular hair cell, a node of Ranvier from mouse dorsal root peripheral nerve, a branched spiny dendrite from rat cerebellum and an unbranched spiny dendrite from rat cerebellum. These images demonstrate the emerging importance of electron tomography as a research tool, particularly for studying the nervous system. (The mitochondria is courtesy of Dr. G. Perkins.) 